\title{
Motivación de los estudiantes universitarios de Ciencias de la Educación según género, cultura religiosa y habilidad social durante la pandemia de COVID-19
}

\author{
Lionel Sánchez-Bolívar \\ Sergio Escalante-González \\ Lindsay Michelle Vázquez \\ Universidad de Granada. España. \\ lionelsanchez@correo.ugr.es \\ sescalante@correo.ugr.es \\ lindsay@correo.ugr.es
}

Recibido: $25 / 2 / 2021$

Aceptado: 22/7/2021

Publicado: 31/1/2022

\section{Resumen}

La situación sanitaria actual ha supuesto un gran reto para el alumnado y el profesorado de todas las etapas educativas. En este sentido, ha sido imperativo adaptar el proceso de enseñanza-aprendizaje a las restricciones sanitarias derivadas de la COVID-19. El objetivo de este análisis es analizar la motivación de los estudiantes de Ciencias de la Educación en relación con su género, su tendencia religiosa y sus habilidades sociales. En la presente investigación participaron un total de 130 estudiantes matriculados en la Universidad de Granada, en los campus de Ceuta y de Melilla $(M=23,38$; DT =6,95), en una investigación ex post facto, de naturaleza cuantitativa, con un diseño descriptivo, correlacional y de corte transversal, que respondieron a la versión española de la escala de motivación situacional de Guay, Vallerand y Blanchard (2000). Entre los resultados se puede destacar que la población más motivada intrínsecamente es la femenina y de religión católica. Respecto a las habilidades sociales, la sociabilidad, la comunicación y la asertividad son las que influyen en el grado de motivación del alumnado. Los resultados de este estudio reflejan la utilidad de analizar la motivación de los universitarios para brindarles una enseñanza más individualizada, destacando que existe una importante necesidad de trabajar sus habilidades sociales.

Palabras clave: aprendizaje individualizado; estudiantes universitarios; género; habilidades sociales; motivación; religión

Resum. Motivació dels estudiants universitaris de Ciències de l'Educació segons gènere, cultura religiosa $i$ habilitat social durant la pandèmia de COVID-19

La situació sanitària actual ha suposat un gran repte per a l'alumnat i el professorat de totes les etapes educatives. En aquest sentit, ha estat imperatiu adaptar el procés d'ensenyament-aprenentatge a les restriccions sanitàries derivades de la COVID-19. En la present investigació hi van participar un total de 130 estudiants universitaris matriculats a la Universitat de Granada, en els campus de Ceuta i de Melilla $(M=23,38$; DT =6,95), en 
un estudi ex post facto, de naturalesa quantitativa, amb un disseny descriptiu, correlacional i de tall transversal, que van respondre a l'adaptació a l'espanyol de l'escala de motivació situacional de Guay, Vallerand i Blanchard (2000). Entre els resultats es pot destacar que la població més motivada intrínsecament és la femenina i de religió catòlica. Pel que fa a les habilitats socials, la sociabilitat, la comunicació i l'assertivitat són les que influeixen en el grau de motivació de l'alumnat. Les dades extretes d'aquest estudi reflecteixen la utilitat d'analitzar la motivació dels universitaris per brindar-los un ensenyament més individualitzat, destacant que hi ha una important necessitat de treballar-ne les habilitats socials.

Paraules clau: aprenentatge individualitzat; estudiants universitaris; gènere; habilitats socials; motivació; religió

Abstract. Motivation of university students in educational sciences according to gender, religious culture, and social skills during the COVID-19 pandemic

The current health situation has posed a significant challenge for students and teachers of all educational stages, as they have had to adapt the teaching-learning process to the health restrictions imposed during the COVID-19 pandemic. The aim of this study is to analyze the motivation of university students in education sciences in relation to their gender, religious tendencies, and social skills. A total of 130 university students enrolled in the University of Granada at the Ceuta and Melilla campuses $(M=23.38$; $S D=6.95)$ participated in this research in an ex post facto quantitative study with a descriptive, correlational, and cross-sectional design. The students responded to the adaptation into Spanish of the Situational Motivation Scale of Guay et al. (2000). Among the results, it can be highlighted that the most intrinsically motivated population is female and Catholic. Regarding social skills, sociability, communication, and assertiveness are the skills that most influence student's degree of motivation. The data drawn from this study demonstrate the usefulness of analyzing students' motivation to provide them with more individualized teaching, as well as the need to work on students' social skills.

Keywords: individualized learning; university students; gender; social skills; motivation; religion
Sumario
1. Introducción
4. Discusión
2. Material y método
5. Conclusiones y limitaciones
3. Resultados
Referencias bibliográficas

\section{Introducción}

El momento actual está marcado por las adaptaciones en el ámbito educativo que han sido impuestas debido a la pandemia de la COVID-19. Entre ellas encontramos el cambio de la modalidad de enseñanza universitaria, que en muchos casos ha pasado de ser presencial a ser en línea. Esto ha repercutido en el estudiantado y ha provocado un aumento del nivel de estrés y de ansiedad, que, por otro lado, no se ha reflejado en los valores de la motivación, aunque sí ha afectado negativamente a la participación de los alumnos (Pasion et al., 2020; Saide y Sheng, 2020). 
Asimismo, la pandemia ha puesto de relieve las diferencias económicas de los estudiantes, lo que, a su vez, ha afectado al proceso de enseñanza-aprendizaje de aquellos que no gozaban de tantos recursos en lo referente a la adquisición de equipos y de programas informáticos o a la contratación de una conexión a internet suficientemente potente. En este punto también cabe destacar que la inexperiencia en el uso de las tecnologías de la información y la comunicación por parte de alumnos y docentes ha influido negativamente en la eficacia del proceso de enseñanza-aprendizaje (Saide y Sheng, 2020).

En relación con lo anterior, en el proceso de enseñanza-aprendizaje es necesaria la influencia positiva del contacto social que permite un aprendizaje colaborativo en el aula y que se ha perdido con el distanciamiento, lo que ha perjudicado a las relaciones entre el alumnado. El cambio inesperado del sistema de enseñanza no ha permitido al profesorado ni a las instituciones adaptarse correctamente, ya que este proceso de actualización requiere tiempo y esfuerzo (Shin y Hickey, 2020; Pasion, 2020).

En este sentido, la motivación capacita a las personas para desarrollarse en diferentes campos, ya que es la que permite alcanzar logros en la vida (Hendrie y Bastacini, 2019). Esto evidencia la necesidad de tener una alta motivación a la hora de conseguir un objetivo como finalizar un grado universitario.

Según Malinauskas y Pozeriene (2020), al comparar los niveles de motivación entre los alumnos universitarios que cursaron clases presenciales y en línea, tanto antes como durante el periodo de pandemia, fue significativo ver que la motivación intrínseca es más alta en estudiantes que asisten a clases en línea y que no se han encontrado diferencias significativas en la variable de género en lo referente a la motivación académica.

Por lo anterior, una alta motivación intrínseca favorece el pensamiento crítico, el desarrollo académico y la capacidad de los estudiantes universitarios para desarrollar estrategias metacognitivas. A la vez, el desarrollo académico se puede predecir usando las variables de motivación, capacidad de pensamiento crítico y nivel de estrés académico (Malinauskas y Pozeriene, 2020; Trigueros et al., 2020).

Por otro lado, un alto nivel de estrés académico se relaciona negativamente con el nivel bajo de motivación y, por tanto, se asocia a un bajo rendimiento estudiantil (Trigueros et al., 2020), pero un nivel de estrés promedio, debido a la ansiedad situacional, contribuye a desarrollar un alto nivel de motivación y favorece la toma de decisiones (Al Majali, 2020).

Asimismo, un alto bienestar psicológico del alumnado también está relacionado con una alta motivación intrínseca. Este bienestar está determinado por la autonomía, la consecución de propósitos, el autoconcepto, el dominio del entorno, el crecimiento personal y las relaciones positivas que se generan gracias a las habilidades sociales. Además, el bienestar no depende del género del alumnado, pero sí influye el curso en el que se encuentre (González Olivares, Navarro, Sánchez-Verdejo y Muelas, 2020; Graf et al., 2020).

En este sentido, Sánchez-Bolívar, Escalante-González y Martínez-Martínez (2021) determinan que no solo es relevante la naturaleza intrínseca o extrín- 
seca, sino también el nivel de interiorización o externalización de la motivación, es decir, de la regulación motivacional. Los autores establecen una intensa relación entre la regulación introyectada y la motivación intrínseca, así como una relación altamente negativa entre la regulación externa y la amotivación del alumnado, por lo que cuanto más externo es el agente motivacional, mayor es el riesgo de que los estudiantes experimenten alienación motivacional.

Las habilidades sociales son capacidades adquiridas que dependen del entorno en el que se desarrollan las personas y que se consiguen debido a un proceso de aprendizaje. Estas habilidades tienen una relación positiva con el clima social en la familia, así como una relación significativa alta entre habilidades sociales y toma de decisiones de los alumnos universitarios (Melgar Begazo, Flores Sotelo, Arévalo Tuesta y Antón de los Santos, 2019; Shi y Du, 2020).

Las habilidades sociales tienen que ver con la capacidad de la persona para relacionarse con su entorno cercano. La falta de habilidades sociales tiene que ver con la inseguridad para interactuar con los demás, ya sea por timidez o por introversión, que también puede provocar temor a la autoridad, miedo al rechazo y dificultad en la comunicación, lo que derivará en una actitud complaciente, influida por la necesidad de aceptarse o por el miedo al rechazo (Javier Napa, Santa María Relaiza, Norabuena Figueroa y Jara Jara, 2019).

En este sentido, autores como Shi y Du (2020) y Graf et al. (2020) exponen que la existencia de un desarrollo en las habilidades sociales implica un aumento de otros factores psicosociales, como la motivación, la inteligencia emocional y las habilidades comunicativas, y cuando aumenta el nivel de estos factores, los estudiantes experimentan un mayor desarrollo social.

Por otro lado, las habilidades sociales están ligadas al bienestar del alumnado, pues estas habilidades permiten reforzar la motivación extrínseca y otras capacidades de adaptación como la empatía, el trabajo cooperativo, el rendimiento o el control de impulsos, todas ellas necesarias para conseguir relaciones exitosas entre estudiantes, aunque no indispensables, puesto que no están relacionadas con el rendimiento académico (Cajas Bravo, Paredes Pérez, Pasquel Loarte y Pasquel Cajas, 2020).

Dada la importancia de la motivación y de las habilidades sociales en el proceso de enseñanza-aprendizaje en esta delicada situación sanitaria, con el presente estudio se pretende describir y analizar qué motivación es predominante en el alumnado universitario, en relación con el género, la religión y las habilidades sociales del mismo.

\section{Material y método}

\subsection{Diseño y población}

Para este estudio de naturaleza cuantitativa y no experimental se empleó un diseño ex post facto de carácter descriptivo, exploratorio, correlacional y de corte transversal. La selección de los participantes se llevó a cabo mediante un muestreo de tipo no probabilístico por conveniencia. 
Participaron un total de 130 estudiantes ( 46 hombres y 84 mujeres), matriculados en los grados de Educación Social $(n=33)$, Educación Infantil $(n=36)$, Educación Primaria $(n=41)$ y Educación Primaria y Ciencias de la Actividad Física y del Deporte $(n=20)$ en los campus de Ceuta y de Melilla, de la Universidad de Granada; con edades comprendidas entre los 18 y los 52 años $(\mathrm{M}=23,38 ; \mathrm{DT}=6,95)$, cuyo alumnado masculino representa el $35,40 \%$ $(n=46)$ y el femenino, el $64,60 \%(n=84)$.

\subsection{Variables e instrumentos}

Para las variables sociodemográficas se empleó un cuestionario ad hoc mediante el que se medía los aspectos siguientes: edad, género (hombre o mujer), campus de estudios (Ceuta o Melilla), religión (cristiana/católica, judía/hebrea, musulmana, hindú y otras), titulación cursada (grados en Educación Infantil, Educación Primaria, Educación Social) y habilidad social (introvertido/extrovertido, pasivo/activo, reactivo/proactivo, asocial/sociable, reservado/comunicativo, pesimista/optimista, indiferente/empático, agresivo/asertivo, sumiso/ dominante, inflexible/adaptativo).

La medición de la motivación se llevó a cabo mediante la adaptación de la escala de motivación situacional (Situational Motivation Scale), creada y validada por Guay, Vallerand y Blanchard (2000) y desarrollada por Martín-Albo, Núnez y Navarro (2009).

El estudio se realizó en una escala Likert que constaba de 16 ítems, cuyas opciones de respuesta iban del 1 al 7 ( 1 equivalía a nada; 2 , a muy poco; 3 , a un poco; 4 , a moderadamente; 5 , a suficiente; 6 , a mucho, y 7 , a totalmente) y que evaluaban cuatro dimensiones: motivación intrínseca (evaluada por los ítems 1, 5, 9 y 13); regulación identificada (evaluada por los ítems 2, 6, 10 y 14); regulación externa (evaluada por los ítems 3, 7, 11 y 15), y amotivación (evaluada por los ítems 4, 8, 12 y 16).

Para la presente investigación este instrumento determinó una fiabilidad de $\alpha=0,88$ para la subescala de motivación intrínseca, $\alpha=0,80$ en la subescala que medía la regulación identificada, $\alpha=0,70$ en la subescala para la medición de la regulación externa y $\alpha=0,76$ para la amotivación.

\subsection{Procedimiento}

Finalizado el diseño del instrumento de recogida de datos se procedió a establecer contacto vía correo electrónico con profesorado coordinador y de relevancia de cada una de las titulaciones de grado del campus de Ceuta y del campus de Melilla. Posteriormente, se acordaba una cita para explicar la investigación y el instrumento al profesorado.

Tras la confirmación positiva se procedía a aplicar el cuestionario a través de Google Forms.

Después del proceso de recogida de datos se procedió a la organización y a la codificación de los cuestionarios. Por último, cabe destacar que esta inves- 
tigación ha seguido las normas éticas del Comité de Ética en Investigación Humana de la Universidad de Granada y de la Declaración de Helsinki.

\subsection{Análisis de los datos}

El análisis de los datos se realizó mediante el programa estadístico IBM SPSS $®$ 25.0. Para los descriptivos básicos se emplearon frecuencias, mientras que para el estudio relacional entre las variables sociodemográficas y las habilidades sociales se emplearon las pruebas paramétricas $t$ de Student para las variables dicotómicas y el ANOVA de un factor para las variables politómicas, puesto que los datos de la muestra seguían una distribución normal y se cumplían los criterios de normalidad y homocedasticidad. Por último, para establecer el grado y el tipo de relación se hizo uso de la correlación bivariada de Pearson.

\section{Resultados}

En este estudio participaron un total de 130 estudiantes, un $64,60 \%(n=84)$ eran mujeres y un $35,40 \%(n=46)$ eran hombres, tal y como se puede observar en la tabla 1, de titulaciones de Ciencias de la Educación de las ciudades transfronterizas de Ceuta y Melilla, cuyos campus universitarios dependen orgánicamente de la Universidad de Granada.

Dentro de esta población, el 49,20\% $(n=64)$ eran cristianos, el 30\% $(n=39)$ eran musulmanes y el $20,80 \%(n=27)$ no manifestaba tendencia religiosa alguna. En cuanto al campus de procedencia, el $82,30 \%(n=107)$ de la muestra procedía del campus de Ceuta, mientras que el $17,70 \%(n=23)$ procedía del campus de Melilla.

Respecto a las habilidades sociales, un $61,50 \%(n=80)$ del alumnado se define como "extrovertido", mientras que un 38,50\% $(n=50)$ lo hace como «introvertido». Por otro lado, el $76,20 \%(n=99)$ se considera socialmente «activo», mientras que el $23,80 \%(n=31)$ tiene una imagen "pasiva» de sí mismo.

Asimismo, el 66,90\% $(n=87)$ se considera "proactivo», mientras que el $33,10 \%(n=43)$ se considera reactivo ante las situaciones sociales. Análogamente, el $87,70 \%(n=114)$ se considera «sociable», aunque existe una pequeña minoría de un $12,30 \%(n=16)$ que se autopercibe como «asocial».

En cuanto a la comunicatividad, el 70,80\% $(n=92)$ manifiesta ser "comunicativo», mientras que el 29,20\% $(n=38)$ reconoce ser «reservado». De igual manera, el $66,90 \%(n=87)$ tiene un pensamiento socialmente "optimista", mientras que el $33,10 \%(n=43)$ es "pesimista".

Tanto la capacidad como la adaptatividad cuentan con los mismos datos. El $88,50 \%(n=155)$ se considera empático y adaptativo, mientras que el $11,50 \%(n=15)$ tiene una imagen de sí mismo de «indiferente» e «inflexible». En cuanto a la reacción emocional, el $78,50 \%(n=102)$ se considera "asertivo" y el $21,50 \%(n=28)$, «agresivo». Asimismo, el $61,50 \%(n=80)$ se considera socialmente «dominante», mientras que el $38,50 \%(n=50)$ se autopercibe como «sumiso». 
Tabla 1. Descriptivos básicos

\begin{tabular}{|c|c|c|c|c|c|c|c|}
\hline & & $n$ & $\%$ & & & $n$ & $\%$ \\
\hline \multirow[t]{2}{*}{ Género } & Mujer & 84 & 64,6 & \multirow[t]{2}{*}{ Comunicatividad } & RESERVADO & 38 & 29,2 \\
\hline & Hombre & 46 & 35,4 & & COMUNICATIVO & 92 & 70,8 \\
\hline \multirow[t]{3}{*}{ Religión } & Cristiana/católica & 64 & 49,2 & \multirow[t]{2}{*}{ Pensamiento } & PESIMISTA & 43 & 33,1 \\
\hline & Musulmana & 39 & 30,0 & & OPTIMISTA & 87 & 66,9 \\
\hline & Ateo & 27 & 20,8 & \multirow[t]{2}{*}{ Capacidad } & INDIFERENTE & 15 & 11,5 \\
\hline \multirow[t]{2}{*}{ Campus } & Campus de Ceuta & 107 & 82,3 & & EMPÁTICO & 115 & 88,5 \\
\hline & Campus de Melilla & 23 & 17,7 & \multirow{2}{*}{$\begin{array}{l}\text { Reacción } \\
\text { emocional }\end{array}$} & AGRESIVO & 28 & 21,5 \\
\hline \multirow[t]{2}{*}{ Actitud } & INTROVERTIDO & 50 & 38,5 & & ASERTIVO & 102 & 78,5 \\
\hline & EXTROVERTIDO & 80 & 61,5 & \multirow[t]{2}{*}{ Control } & SUMISO & 50 & 38,5 \\
\hline \multirow{2}{*}{$\begin{array}{l}\text { Actividad } \\
\text { social }\end{array}$} & PASIVO & 31 & 23,8 & & DOMINANTE & 80 & 61,5 \\
\hline & ACTIVO & 99 & 76,2 & \multirow[t]{3}{*}{ Adaptatividad } & INFLEXIBLE & 15 & 11,5 \\
\hline \multirow{2}{*}{$\begin{array}{l}\text { Reacción } \\
\text { social }\end{array}$} & REACTIVO & 43 & 33,1 & & ADAPTATIVO & 115 & 88,5 \\
\hline & PROACTIVO & 87 & 66,9 & & & & \\
\hline Sociabilidad & ASOCIAL & 16 & 12,3 & Sociabilidad & SOCIABLE & 114 & 87,7 \\
\hline
\end{tabular}

Fuente: elaboración propia.

En cuanto a la relación entre la motivación del alumnado y el género, tal y como se aprecia en la tabla 2 , se han hallado diferencias significativas a nivel estadístico entre la «motivación intrínseca» y el género $(p=0,000)$. En este sentido, las mujeres $(M=5,46 ; \mathrm{DT}=1,33)$ se encuentran más motivadas intrínsecamente que los hombres $(M=4,50$; $D T=1,56)$, existiendo una diferencia de medias entre géneros de casi un punto. De forma análoga, los hombres cuentan con niveles superiores de "amotivación» $(\mathrm{M}=2,27 ; \mathrm{DT}=1,23)$ a los de las mujeres $(M=1,71$; DT 1,01), siendo estadísticamente significativas estas diferencias $(p=0,005)$.

En lo que respecta a la relación entre la cultura religiosa y la motivación de la población, se han identificado diferencias estadísticamente significativas entre la "religión" y la "motivación intrínseca» $(p=0,044)$. En este sentido, el alumnado cristiano $(\mathrm{M}=5,31$; $\mathrm{DT}=1,34)$ cuenta con niveles medios de "motivación intrínseca» superiores al alumnado musulmán $(\mathrm{M}=5,24 ; \mathrm{DT}=1,61)$ y a las del alumnado ateo $(\mathrm{M}=4,49 ; \mathrm{DT}=1,49)$. 
Tabla 2. Relación entre el género, la religión y la titulación y la motivación del alumnado

\begin{tabular}{|c|c|c|c|c|c|c|c|c|c|}
\hline & & M & DT & \multicolumn{3}{|l|}{$p$} & M & DT & $p$ \\
\hline \multicolumn{10}{|c|}{ Género } \\
\hline \multirow[t]{2}{*}{$\mathrm{Ml}$} & Mujer & 5,46 & 1,33 & \multirow[t]{2}{*}{0,000} & \multirow[t]{2}{*}{ RE } & Mujer & 3,39 & 1,55 & \multirow[t]{2}{*}{0,293} \\
\hline & Hombre & 4,50 & 1,56 & & & Hombre & 3,65 & 1,21 & \\
\hline \multirow[t]{2}{*}{ RI } & Mujer & 6,20 & 1,00 & \multirow[t]{2}{*}{0,051} & \multirow[t]{2}{*}{ AM } & Mujer & 1,71 & 1,01 & \multirow[t]{2}{*}{0,005} \\
\hline & Hombre & 5,85 & 0,90 & & & Hombre & 2,27 & 1,23 & \\
\hline \multicolumn{10}{|c|}{ Religión } \\
\hline \multirow[t]{3}{*}{ Ml } & Cristiana/católica & 5,31 & 1,34 & \multirow[t]{3}{*}{0,044} & \multirow[t]{3}{*}{ RE } & Cristiana/católica & 3,54 & 1,50 & \multirow[t]{3}{*}{0,073} \\
\hline & Musulmana & 5,24 & 1,61 & & & Musulmana & 3,75 & 1,44 & \\
\hline & Ateo & 4,49 & 1,49 & & & Ateo & 2,94 & 1,17 & \\
\hline \multirow[t]{3}{*}{$\mathrm{RI}$} & Cristiana/católica & 6,22 & 0,92 & \multirow[t]{3}{*}{0,196} & \multirow[t]{3}{*}{ AM } & Cristiana/católica & 1,83 & 1,13 & \multirow[t]{3}{*}{0,217} \\
\hline & Musulmana & 6,00 & 1,13 & & & Musulmana & 1,79 & 1,12 & \\
\hline & Ateo & 5,83 & 0,83 & & & Ateo & 2,24 & 1,07 & \\
\hline \multicolumn{10}{|c|}{ Titulación } \\
\hline \multirow[t]{4}{*}{ Ml } & $\begin{array}{l}\text { Grado en Educación } \\
\text { Infantil }\end{array}$ & 5,53 & 1,37 & \multirow[t]{4}{*}{0,055} & \multirow[t]{4}{*}{ RE } & $\begin{array}{l}\text { Grado en Educación } \\
\text { Infantil }\end{array}$ & 3,53 & 1,53 & \multirow[t]{4}{*}{0,405} \\
\hline & $\begin{array}{l}\text { Grado en Educación } \\
\text { Primaria }\end{array}$ & 4,70 & 1,58 & & & $\begin{array}{l}\text { Grado en Educación } \\
\text { Primaria }\end{array}$ & 3,52 & 1,53 & \\
\hline & $\begin{array}{l}\text { Grado en Educación } \\
\text { Social }\end{array}$ & 4,98 & 1,61 & & & $\begin{array}{l}\text { Grado en Educación } \\
\text { Social }\end{array}$ & 3,66 & 1,29 & \\
\hline & $\begin{array}{l}\text { Grado en Educación } \\
\text { Primaria y Ciencias de } \\
\text { la Actividad Física y del } \\
\text { Deporte }\end{array}$ & 5,48 & 0,94 & & & $\begin{array}{l}\text { Grado en Educación } \\
\text { Primaria y Ciencias de } \\
\text { la Actividad Física y del } \\
\text { Deporte }\end{array}$ & 2,99 & 1,30 & \\
\hline \multirow[t]{4}{*}{$\mathrm{RI}$} & $\begin{array}{l}\text { Grado en Educación } \\
\text { Infantil }\end{array}$ & 6,42 & 0,88 & \multirow[t]{4}{*}{0,051} & \multirow[t]{4}{*}{ AM } & $\begin{array}{l}\text { Grado en Educación } \\
\text { Infantil }\end{array}$ & 1,65 & 0,96 & \multirow[t]{4}{*}{0,334} \\
\hline & $\begin{array}{l}\text { Grado en Educación } \\
\text { Primaria }\end{array}$ & 5,85 & 1,13 & & & $\begin{array}{l}\text { Grado en Educación } \\
\text { Primaria }\end{array}$ & 2,06 & 1,18 & \\
\hline & $\begin{array}{l}\text { Grado en Educación } \\
\text { Social }\end{array}$ & 5,91 & 0,97 & & & $\begin{array}{l}\text { Grado en Educación } \\
\text { Social }\end{array}$ & 2,05 & 1,25 & \\
\hline & $\begin{array}{l}\text { Grado en Educación } \\
\text { Primaria y Ciencias de } \\
\text { la Actividad Física y del } \\
\text { Deporte }\end{array}$ & 6,18 & 0,63 & & & $\begin{array}{l}\text { Grado en Educación } \\
\text { Primaria y Ciencias de } \\
\text { la Actividad Física y del } \\
\text { Deporte }\end{array}$ & 1,81 & 1,05 & \\
\hline
\end{tabular}

Nota: MI: Motivación Intrínseca; RI: Regulación Identificada; RE: Regulación Externa; AM: Amotivación. Fuente: elaboración propia.

Por otro lado, en la relación entre las habilidades sociales del alumnado y la motivación, se han identificado diferencias estadísticamente significativas entre la sociabilidad del alumnado y la motivación intrínseca $(p=0,044)$, contando el alumnado asocial $(\mathrm{M}=4,42$; $\mathrm{DT}=1,67)$ con un nivel de motivación intrínseca inferior al del alumnado social $(M=5,22 ; \mathrm{DT}=1,67)$. 
Tabla 3. Relación entre las habilidades sociales y la motivación del alumnado

\begin{tabular}{|c|c|c|c|c|c|c|c|c|c|}
\hline $\begin{array}{l}\text { Dimen- } \\
\text { sión }\end{array}$ & Habilidad social & M & DT & $p$ & $\begin{array}{l}\text { Dimen- } \\
\text { sión }\end{array}$ & Habilidad social & M & DT & $p$ \\
\hline \multicolumn{10}{|c|}{ Habilidades sociales: Actitud social } \\
\hline \multirow[t]{2}{*}{$\mathrm{Ml}$} & INTROVERTIDO & 4,85 & 1,50 & \multirow[t]{2}{*}{0,101} & \multirow[t]{2}{*}{$\mathrm{RE}$} & INTROVERTIDO & 3,63 & 1,35 & \multirow[t]{2}{*}{0,346} \\
\hline & EXTROVERTIDO & 5,29 & 1,45 & & & EXTROVERTIDO & 3,38 & 1,49 & \\
\hline \multirow[t]{2}{*}{ RI } & INTROVERTIDO & 6,01 & 0,99 & \multirow[t]{2}{*}{0,532} & \multirow[t]{2}{*}{ AM } & INTROVERTIDO & 1,98 & 1,06 & \multirow[t]{2}{*}{0,553} \\
\hline & EXTROVERTIDO & 6,12 & 0,98 & & & EXTROVERTIDO & 1,86 & 1,17 & \\
\hline \multicolumn{10}{|c|}{ Habilidades sociales: Actividad } \\
\hline \multirow[t]{2}{*}{ MI } & PASIVO & 4,81 & 1,61 & \multirow[t]{2}{*}{0,190} & \multirow[t]{2}{*}{$\mathrm{RE}$} & PASIVO & 3,44 & 1,30 & \multirow[t]{2}{*}{0,876} \\
\hline & ACTIVO & 5,21 & 1,43 & & & ACTIVO & 3,49 & 1,49 & \\
\hline \multirow[t]{2}{*}{$\mathrm{RI}$} & PASIVO & 6,00 & 1,02 & \multirow[t]{2}{*}{0,635} & \multirow[t]{2}{*}{ AM } & PASIVO & 2,06 & 1,15 & \multirow[t]{2}{*}{0,394} \\
\hline & ACTIVO & 6,10 & 0,97 & & & ACTIVO & 1,86 & 1,11 & \\
\hline \multicolumn{10}{|c|}{ Habilidades sociales: Reacción social } \\
\hline \multirow[t]{2}{*}{$\mathrm{Ml}$} & REACTIVO & 5,08 & 1,54 & \multirow[t]{2}{*}{0,839} & \multirow[t]{2}{*}{$\mathrm{RE}$} & REACTIVO & 3,32 & 1,48 & \multirow[t]{2}{*}{0,378} \\
\hline & PROACTIVO & 5,14 & 1,46 & & & PROACTIVO & 3,56 & 1,42 & \\
\hline \multirow[t]{2}{*}{$\mathrm{RI}$} & REACTIVO & 6,15 & 0,90 & \multirow[t]{2}{*}{0,524} & \multirow[t]{2}{*}{ AM } & REACTIVO & 1,83 & 1,09 & \multirow[t]{2}{*}{0,597} \\
\hline & PROACTIVO & 6,03 & 1,02 & & & PROACTIVO & 1,94 & 1,14 & \\
\hline & & & abilida & es soci & les: Soc & labilidad & & & \\
\hline $\mathrm{Ml}$ & ASOCIAL & 4,42 & 1,67 & 0,044 & $\mathrm{RE}$ & ASOCIAL & 3,56 & 1,51 & 0,805 \\
\hline & SOCIABLE & 5,22 & 1,43 & & & SOCIABLE & 3,47 & 1,44 & \\
\hline $\mathrm{RI}$ & ASOCIAL & 5,47 & 1,30 & 0,055 & AM & ASOCIAL & 2,41 & 1,05 & 0,056 \\
\hline & SOCIABLE & 6,16 & 0,90 & & & SOCIABLE & 1,84 & 1,12 & \\
\hline & & $\mathrm{Hab}$ & ilidade & sociale & s: Comu & nicatividad & & & \\
\hline $\mathrm{Ml}$ & RESERVADO & 4,84 & 1,58 & 0,161 & RE & RESERVADO & 3,69 & 1,43 & 0,283 \\
\hline & COMUNICATIVO & 5,24 & 1,43 & & & COMUNICATIVO & 3,39 & 1,44 & \\
\hline RI & RESERVADO & 5,80 & 1,13 & 0,042 & AM & RESERVADO & 2,22 & 1,24 & 0,037 \\
\hline & COMUNICATIVO & 6,18 & 0,89 & & & COMUNICATIVO & 1,77 & 1,05 & \\
\hline & & & bilidac & es socit & les: Pen & samiento & & & \\
\hline $\mathrm{Ml}$ & PESIMISTA & 4,78 & 1,49 & 0,065 & RE & PESIMISTA & 3,41 & 1,54 & 0,715 \\
\hline & OPTIMISTA & 5,29 & 1,46 & & & OPTIMISTA & 3,51 & 1,40 & \\
\hline RI & PESIMISTA & 5,90 & 0,97 & 0,159 & $\mathrm{AM}$ & PESIMISTA & 2,07 & 1,12 & 0,243 \\
\hline & OPTIMISTA & 6,16 & 0,98 & & & OPTIMISTA & 1,82 & 1,12 & \\
\hline & & & labilid & des soc & ales: $\mathrm{Ca}$ & sacidad & & & \\
\hline $\mathrm{Ml}$ & INDIFERENTE & 4,20 & 1,98 & 0,067 & $\mathrm{RE}$ & INDIFERENTE & 3,45 & 1,53 & 0,935 \\
\hline & EMPÁTICO & 5,24 & 1,37 & & & EMPÁTICO & 3,48 & 1,44 & \\
\hline
\end{tabular}




\begin{tabular}{|c|c|c|c|c|c|c|c|c|c|}
\hline $\begin{array}{l}\text { Dimen- } \\
\text { sión }\end{array}$ & Habilidad social & M & DT & $p$ & $\begin{array}{l}\text { Dimen- } \\
\text { sión }\end{array}$ & Habilidad social & M & DT & $p$ \\
\hline \multirow[t]{2}{*}{ RI } & INDIFERENTE & 5,93 & 1,01 & \multirow[t]{2}{*}{0,558} & \multirow[t]{2}{*}{ AM } & INDIFERENTE & 2,30 & 0,94 & \multirow[t]{2}{*}{0,149} \\
\hline & EMPÁTICO & 6,09 & 0,98 & & & EMPÁTICO & 1,85 & 1,14 & \\
\hline \multicolumn{10}{|c|}{ Habilidades sociales: Reacción emocional } \\
\hline \multirow[t]{2}{*}{$\mathrm{Ml}$} & AGRESIVO & 4,37 & 1,65 & \multirow[t]{2}{*}{0,002} & \multirow[t]{2}{*}{$\mathrm{RE}$} & AGRESIVO & 3,50 & 1,51 & \multirow[t]{2}{*}{0,930} \\
\hline & ASERTIVO & 5,33 & 1,37 & & & ASERTIVO & 3,47 & 1,43 & \\
\hline \multirow[t]{2}{*}{ RI } & AGRESIVO & 5,79 & 0,98 & \multirow[t]{2}{*}{0,079} & \multirow[t]{2}{*}{ AM } & AGRESIVO & 2,48 & 1,13 & \multirow[t]{2}{*}{0,002} \\
\hline & ASERTIVO & 6,15 & 0,96 & & & ASERTIVO & 1,75 & 1,07 & \\
\hline \multicolumn{10}{|c|}{ Habilidades sociales: Control } \\
\hline \multirow[t]{2}{*}{$\mathrm{Ml}$} & SUMISO & 5,23 & 1,23 & \multirow[t]{2}{*}{0,495} & \multirow[t]{2}{*}{$\mathrm{RE}$} & SUMISO & 3,40 & 1,39 & \multirow[t]{2}{*}{0,602} \\
\hline & DOMINANTE & 5,05 & 1,62 & & & DOMINANTE & 3,53 & 1,48 & \\
\hline \multirow[t]{2}{*}{$\mathrm{RI}$} & SUMISO & 6,14 & 0,79 & \multirow[t]{2}{*}{0,570} & \multirow[t]{2}{*}{ AM } & SUMISO & 1,82 & 0,93 & \multirow[t]{2}{*}{0,468} \\
\hline & DOMINANTE & 6,03 & 1,08 & & & DOMINANTE & 1,96 & 1,23 & \\
\hline \multicolumn{10}{|c|}{ Habilidades sociales: Adaptatividad } \\
\hline \multirow[t]{2}{*}{$\mathrm{Ml}$} & INFLEXIBLE & 4,87 & 1,45 & \multirow[t]{2}{*}{0,484} & \multirow[t]{2}{*}{$\mathrm{RE}$} & INFLEXIBLE & 4,05 & 1,26 & \multirow[t]{2}{*}{0,030} \\
\hline & ADAPTATIVO & 5,15 & 1,49 & & & ADAPTATIVO & 3,40 & 1,45 & \\
\hline \multirow[t]{2}{*}{ RI } & INFLEXIBLE & 6,12 & 0,98 & \multirow[t]{2}{*}{0,855} & \multirow[t]{2}{*}{ AM } & INFLEXIBLE & 2,37 & 1,18 & \multirow[t]{2}{*}{0,091} \\
\hline & ADAPTATIVO & 6,07 & 0,98 & & & ADAPTATIVO & 1,85 & 1,11 & \\
\hline
\end{tabular}

Nota: MI: Motivación Intrínseca; RI: Regulación Identificada; RE: Regulación Externa; AM: Amotivación. Fuente: elaboración propia.

En lo que respecta a la comunicatividad del alumnado y a su nivel de motivación, se han hallado diferencias significativas a nivel estadístico en la regulación identificada $(p=0,042)$, siendo el alumnado comunicativo $(M=6,18$; $\mathrm{DT}=0,89)$ el que manifiesta niveles de motivación superiores al reservado $(\mathrm{M}=5,80 ; \mathrm{DT}=1,13)$.

De igual manera, existen diferencias estadísticamente relevantes en amotivación $(p=0.037)$, y el estudiantado reservado $(\mathrm{M}=2,22$; $\mathrm{DT}=1,24)$ manifiesta niveles de amotivación más elevados que el comunicativo $(M=1,77$; $\mathrm{DT}=1,05)$.

Asimismo, se han identificado diferencias estadísticamente significativas entre la reacción emocional del alumnado y su nivel de motivación intrínseca $(p=0,002)$. Los niveles más altos se sitúan en el alumnado asertivo $(M=5,33$; $\mathrm{DT}=1,37)$ y los más bajos, en el estudiantado agresivo $(\mathrm{M}=4,37$; $\mathrm{DT}=1,65)$.

De forma opuesta, se han hallado diferencias relevantes a nivel estadístico en amotivación $(p=0,002)$, siendo el alumnado agresivo $(\mathrm{M}=2,48 ; \mathrm{DT}=1,13)$ el más amotivado, y el asertivo $(\mathrm{M}=1,75 ; \mathrm{DT}=1,07)$ el que cuenta con una menor amotivación.

En cuanto a la relación entre las variables, tal y como figura en la tabla 4, la motivación intrínseca (MI) correlaciona de forma positiva con todas las 
habilidades sociales, siendo moderada y significativa la correlación entre la motivación intrínseca y la reacción emocional del alumnado $(p=0,396)$, por lo que cuanto más asertivo es el alumnado, más motivado intrínsecamente está.

Asimismo, la motivación intrínseca mantiene una alta correlación positiva y significativa con la regulación identificada $(p=0,673)$, así como una correlación negativa, moderada y significativa con la amotivación $(p=-0,542)$.

En cuanto a la amotivación, correlaciona positivamente con la reacción social $(p=0,047)$, aunque no de forma significativa, y con el control social $(p=0,064)$. Respecto al resto de habilidades sociales, la amotivación correlaciona negativamente con todas las habilidades sociales, $y$ de forma significativa con la reacción emocional $(\mathrm{p}=-0,0270)$, siendo esta correlación moderada, lo que significa que cuanto más asertivo es el alumnado, menor es el nivel de amotivación y viceversa.

De igual manera, la amotivación correlaciona de forma negativa y moderada con la motivación intrínseca $(p=-0,542)$ y con la regulación identificada $(p=-0,551)$, y de forma positiva, y moderada, con la regulación externa $(p=0,410)$.

Tabla 4. Matriz de correlaciones entre las habilidades sociales y la motivación del alumnado

\begin{tabular}{|c|c|c|c|c|c|c|c|c|c|c|c|c|c|c|}
\hline & I-E & P-A & $R-P$ & A-S & R-C & P-O & I-E & $A-A$ & S-D & $\mathrm{I}-\mathrm{A}$ & MI & RI & RE & AM \\
\hline I-E & 1 & $0,337^{* *}$ & $0,184^{*}$ & $0,185^{*}$ & $0,396^{* *}$ & 0,049 & $0,209^{*}$ & 0,009 & $0,285^{*}$ & 0,160 & 0,144 & 0,055 & $-0,083$ & $-0,053$ \\
\hline P-A & $0,337^{* *}$ & 1 & $0,259^{* *}$ & $0,340^{* *}$ & $0,394^{* *}$ & $0,182^{*}$ & $0,363^{* *}$ & 0,146 & $0,263^{* *}$ & 0,137 & 0,116 & 0,042 & 0,014 & $-0,075$ \\
\hline$R \cdot P$ & $0,184^{*}$ & $0,259^{* *}$ & 1 & 0,035 & $0,231^{* *}$ & $0,235^{* *}$ & $0,258^{* *}$ & 0,109 & 0,083 & 0,104 & 0,018 & $-0,056$ & 0,078 & 0,047 \\
\hline A-S & $0,185^{*}$ & $0,340^{* \prime}$ & 0,035 & 1 & $0,428^{* *}$ & $0,334^{* *}$ & $0,304^{* *}$ & 0,089 & $0,281^{* *}$ & $0,231^{* *}$ & $0,177^{*}$ & $0,233^{* *}$ & $-0,022$ & $-0,168$ \\
\hline $\mathrm{R}-\mathrm{C}$ & 0,396 & $0,394^{* *}$ & $0,231^{* *}$ & $0,428^{* *}$ & 1 & $0,195^{*}$ & $0,244^{*}$ & 0,116 & 0,083 & $0,191^{*}$ & 0,124 & $0,179^{*}$ & $-0,095$ & $-0,183^{*}$ \\
\hline P-O & 0,049 & $0,182^{*}$ & $0,235^{* *}$ & $0,334^{* *}$ & $0,195^{*}$ & 1 & $0,207^{*}$ & $0,268^{* *}$ & 0,116 & 0,155 & 0,162 & 0,124 & 0,032 & $-0,103$ \\
\hline I-E & $0,209^{*}$ & $0,363^{* *}$ & $0,258^{* *}$ & $0,304^{* *}$ & $0,244^{* *}$ & $0,207^{*}$ & 1 & $0,338^{* *}$ & $0,209^{*}$ & $0,322^{* *}$ & $0,225^{* *}$ & 0,052 & 0,007 & $-0,127$ \\
\hline A-A & 0,009 & 0,146 & 0,109 & 0,089 & 0,116 & $0,268^{* *}$ & $0,338^{* *}$ & 1 & 0,009 & 0,396 & $0,268^{* *}$ & 0,155 & $-0,008$ & $-0,270^{\prime \prime}$ \\
\hline S-D & $0,285^{* *}$ & $0,263^{*}$ & 0,083 & $0,281^{* *}$ & 0,083 & 0,116 & $0,209^{*}$ & 0,009 & 1 & 0,110 & $-0,057$ & $-0,050$ & 0,046 & 0,064 \\
\hline I-A & 0,160 & 0,137 & 0,104 & $0,231^{* *}$ & $0,191^{*}$ & 0,155 & $0,322^{* *}$ & $0,396^{* *}$ & 0,110 & 1 & 0,062 & $-0,016$ & $-0,144$ & $-0,149$ \\
\hline $\mathrm{Ml}$ & 0,144 & 0,116 & 0,018 & $0,177^{*}$ & 0,124 & 0,162 & $0,225^{*}$ & $0,268^{* *}$ & $-0,057$ & 0,062 & 1 & $0,673^{* *}$ & $-0,159$ & $-0,542^{* *}$ \\
\hline $\mathrm{Rl}$ & 0,055 & 0,042 & $-0,056$ & $0,233^{* *}$ & $0,179^{*}$ & 0,124 & 0,052 & 0,155 & $-0,050$ & $-0,016$ & $0,673^{* *}$ & 1 & $-0,206^{*}$ & $-0,551^{* *}$ \\
\hline $\mathrm{RE}$ & $-0,083$ & 0,014 & 0,078 & $-0,022$ & $-0,095$ & 0,032 & 0,007 & $-0,008$ & 0,046 & $-0,144$ & $-0,159$ & $-0,206^{*}$ & 1 & $0,410^{*}$ \\
\hline $\mathrm{AM}$ & $-0,053$ & $-0,075$ & 0,047 & $-0,168$ & $-0,183^{*}$ & $-0,103$ & $-0,127$ & $-0,270^{* *}$ & 0,064 & $-0,149$ & $-0,542^{+*}$ & $-0,551^{* *}$ & $0,410^{* *}$ & 1 \\
\hline
\end{tabular}

** La correlación es significativa al nivel 0,01 (bilateral).

* La correlación es significante al nivel 0,05 (bilateral).

Fuente: elaboración propia. 


\section{Discusión}

En este estudio se ha procedido a analizar el grado de motivación entre universitarios matriculados en grados de Ciencias de la Educación. La población estudiantil que manifiesta un mayor nivel de motivación intrínseca es la femenina.

Esto se corresponde con lo manifestado por Manzano-Sánchez, PostigoPérez, Gómez-López y Valero-Valenzuela (2020), que defienden que la motivación de las mujeres es de naturaleza intrínseca, basada en la autonomía personal, y que la motivación masculina es de carácter extrínseco, centrada en la tarea. Esto refuerza lo expuesto por Hoyland et al. (2020), que manifiestan que los hombres tienen una alta motivación en relación con la autoeficacia como líderes y las mujeres no.

En contraposición, Malchrowicz-Mośko, Zarębski y Kwiatkowski (2020) defienden que la motivación intrínseca se modula en hombres y mujeres en función de la actividad en la que se centren. En este sentido, MalchrowiczMośko et al. (2020) exponen que la motivación intrínseca masculina es superior en referencia a la competición y el desarrollo de habilidades, mientras que la motivación intrínseca es superior en mujeres cuando se centra en actitudes prosociales como la amistad y el trabajo en equipo.

En cuanto a la religión, tal y como apuntan Sokolovskaya et al. (2020), existen diferencias en los niveles de motivación, de modo que el alumnado musulmán muestra un nivel más bajo de motivación intrínseca que el resto de estudiantes. Todo ello casa con lo expuesto por Vishkin et al. (2019).

Tal y como se ha demostrado con este estudio, existe una relación positiva entre la motivación intrínseca y las habilidades sociales de los estudiantes universitarios de Ciencias de la Educación. De acuerdo con lo que apuntan Tseng, Yi y Yeh (2019) y Fung y Chung (2019), la motivación y la autorregulación influyen sobre las habilidades sociales y ejercen un efecto positivo en el aprendizaje de los estudiantes y en su futura incorporación laboral.

Relacionado con lo anterior, Morrison et al. (2020) añaden que la motivación mejora las habilidades sociales y el desempeño social del alumnado y viceversa, es decir, que una mayor competencia social aumenta, también, la motivación de los estudiantes.

En este sentido, cabe destacar que la desregulación motivacional tiene un efecto negativo en la regulación social, es decir, la falta de regulación motivacional conlleva una desregulación de las habilidades sociales. En este sentido, un alto nivel de motivación provoca una mejora sustancial en el nivel de habilidades sociales de los estudiantes (Neuhaus, Webb y Bernier, 2019).

En el caso de estudiantes universitarios de Ciencias de la Educación, la alienación motivacional o amotivación se relaciona con una falta de comunicatividad, lo que refuerza lo manifestado por Fung y Chung (2019), que concluían que la motivación más elevada se relacionaba con un mayor nivel de destreza social, cosa que aumentaba y mejoraba la comunicatividad del alumnado. 
Los resultados de esta investigación, en consonancia con lo manifestado por Sánchez et al. (2021), ponen de relieve que la motivación de los estudiantes que se están formando como futuros docentes, así como su perfil académicoprofesional, es fundamental, puesto que va a influir en su futura actividad educativa. En este sentido, cuanto mayor sea el nivel de competencias profesionales, personales, sociales, psicológicas y emocionales que los universitarios desarrollen en su etapa estudiantil, más capacitados van a estar para afrontar su futura actividad docente y mayor va a ser la calidad de la enseñanza que aportarán a su futuro alumnado.

Este estudio ha demostrado que la motivación intrínseca se relaciona con una mayor asertividad, mientras que una amotivación alta se relaciona con la tendencia del alumnado a la agresividad. Estos datos se encuentran en consonancia con lo manifestado por Van der Stouwe et al. (2018), que defienden que la motivación es un predictor de la empatía, por lo que una alta motivación se relaciona con una mayor capacidad empática, lo que reduce la hostilidad del alumnado. Van der Stouwe et al. (2018) postulan que esta mayor capacidad empática y la alta motivación contribuyen a facilitar el desarrollo de una mayor capacidad de resolución de conflictos.

\section{Conclusiones y limitaciones}

La principal conclusión que se extrae de este estudio es que el alumnado femenino y católico se encuentra intrínsecamente más motivado que el masculino y que el musulmán o el ateo.

En cuanto a la habilidad social de los estudiantes, el alumnado sociable cuenta con mayor nivel de motivación intrínseca que el asocial. De igual manera, el alumnado reservado cuenta con un nivel más elevado de amotivación que el de los estudiantes más comunicativos a nivel social, existiendo una correlación negativa leve entre la amotivación y la sociabilidad del alumnado, por lo que cuanto más sociable es el alumnado, menor es su amotivación.

Por otro lado, los estudiantes con tendencia a la asertividad cuentan con un mayor nivel de motivación intrínseca que el alumnado con tendencias agresivas. En contraposición, el alumnado agresivo está más amotivado que el estudiantado asertivo. Existe una correlación negativa y moderada entre la asertividad y la amotivación, por lo que cuanto más asertivo es el alumnado, menor es el nivel de alienación motivacional que experimenta, mientras que cuanto más agresivo es, más amotivado está.

Este estudio ha puesto de manifiesto la utilidad de analizar la motivación del alumnado para brindarle una enseñanza más individualizada. De igual manera, se ha concluido que existe una importante necesidad de trabajar sobre las habilidades sociales de los estudiantes, sobre todo en la reacción social, dado que la alienación motivacional puede ser prevenida mediante el entrenamiento de la asertividad.

La principal limitación de este estudio ha sido la recogida de datos, puesto que, al establecerse restricciones sanitarias a causa de la pandemia motivada 
por la COVID-19, ha sido sumamente complicado acceder a una muestra más numerosa. Esta limitación abre una posible línea de investigación futura, que debería realizarse ampliando la muestra y analizando los datos desde un enfoque más predictivo.

De igual manera, otra línea de investigación posible consistiría en desarrollar esta misma investigación en un escenario pospandemia, para evaluar y comparar tanto la influencia de diversos escenarios sociosanitarios como las diferentes formas de enseñanza-aprendizaje (presencial, virtual, semipresencial, etc.) sobre la motivación y las habilidades sociales del alumnado.

\section{Referencias bibliográficas}

Al Majali, S. (2020). Positive Anxiety and its Role in Motivation and Achievements among University Students. International Journal of Instruction, 13(4), 975-986. $<$ https://doi.org/10.29333/iji.2020.13459a>

Cajas Bravo, V.; Paredes Pérez, M.A.; Pasquel loarte, L. y Pasquel Cajas, A.F. (2020). Habilidades sociales en Engagement y desempeño académico en estudiantes universitarios.Comuni@cción: Revista de Investigación en Comunicación y Desarrollo, 11(1), 77-88. <https://doi.org/10.33595/2226-1478.11.1.405>

Dawson, L.L. (2018). Debating the Role of Religion in the Motivation of Religious Terrorism. Nordic Journal of Religion and Society, 31(2), 98-117. $<$ https://doi.org/10.18261/issn.1890-7008-2018-02-02>

Díaz-López, L.; TARAngo, J. y Refugio Romo-González, J. (2020). Realidad virtual en procesos de aprendizaje en estudiantes universitarios: Motivación e interés para despertar vocaciones científicas. Cuadernos de Documentación Multimedia, 31. <https://doi.org/10.5209/cdmu.68958>

Fung, W. y Chung, K.K. (2019). The direct and indirect relationships among kindergarten children's social mastery motivation, receptive vocabulary, and socioemotional skills. Current Psychology, 40. $<$ https://doi.org/10.1007/s12144-019-00523-3>

González Olivares, Á.L.; Navarro, Ó.; Sánchez-Verdejo, F.J. y Muelas, Á. (2020). Psychological Well-Being and Intrinsic Motivation: Relationship in Students Who Begin University Studies at the School of Education in Ciudad Real. Frontiers in Psychology, 11, 2054. $<$ https://doi.org/10.3389/fpsyg.2020.02054>

Goolsby, J.; Alvord, M.K. y Rich, B.A. (2019). Impact of Child and Parent Motivation on Social Skills Training Outcome. Child \& Family Behavior Therapy, 41(1), $32-46$. <https://doi.org/10.1080/07317107.2019.1571774>

Graf, J.; Loda, T.; Zipfel, S.; Wosnik, A.; Mohr, D. y Herrmann-Werner, A. (2020). Communication skills of medical students: Survey of self-and external perception in a longitudinally based trend study. BMC Medical Education, 20(1), 149. $<$ https://doi.org/10.1186/s12909-020-02049-w>

Guay, F.; Vallerand, R. y Blanchard, C. (2000). On the Assessment of Situational Intrinsic and Extrinsic Motivation: The Situational Motivation Scale (SIMS). Motivation and Emotion, 24(3), 175-213. $<$ http://dx.doi.org/10.1023/a:1005614228250> 
Hendrie Kupczyszyn, K.N. y Bastacini, M.D.C. (2019). Autorregulación en estudiantes universitarios: Estrategias de aprendizaje, motivación y emociones. Revista Educación, 44(1), 29. <https://doi.org/10.15517/revedu.v44i1.37713>

Hoyland, T.; Psychogios, A.; Epitropaki, O.; Damiani, J.; Mukhuty, S. y PriestNALL, C. (2021). A two-nation investigation of leadership self-perceptions and motivation to lead in early adulthood: The moderating role of gender and socioeconomic status. Leadership \& Organization Development Journal [edición en línea previa a la publicación impresa].

<https://doi.org/10.1108/lodj-03-2020-0112>

Javier Napa, A.J.; Santa María Relaiza, H.R.; Norabuena Figueroa, R.P. y Jara JARA, N. (2019). Acción tutorial para el desarrollo de las habilidades sociales en estudiantes universitarios. Propósitos y Representaciones, 7(1), 185. <https://doi.org/10.20511/pyr2019.v7n1.261>

Malchrowicz-Mośko, E.; ZaręBski, P. y Kwiatkowski, G. (2020). What Triggers Us to Be Involved in Martial Arts?: Relationships between Motivations and Gender, Age and Training Experience. Sustainability, 12(16), 6567. $<$ https://doi.org/10.3390/su12166567>

Malinauskas, R.K. y Pozeriene, J. (2020). Academic Motivation among Traditional and Online University Students. European Journal of Contemporary Education, 9(3). <https://doi.org/10.13187/ejced.2020.3.584>

Manzano-Sánchez, D.; Postigo-Pérez, L.; Gómez-López, M. y Valero-Valenzuela, A. (2020). Study of the Motivation of Spanish Amateur Runners Based on Training Patterns and Gender. International Journal of Environmental Research and Public Health, 17(21), 8185. <https://doi.org/10.3390/ijerph17218185>

Martín-Albo, J.; NúÑez, J. y Navarro, J. (2009). Validation of the Spanish Version of the Situational Motivation Scale (EMSI) in the Educational Context. The Spanish Journal of Psychology, 12(2), 799-807. <https://doi.org/10.1017/s113874160000216x>

Melgar Begazo, A.E.; Flores Sotelo, W.S.; Arévalo Tuesta, J.A. y Antón de los SAntos, P.J. (2019). Tecnologías educativas, habilidades sociales y la toma de decisiones en estudiantes universitarios. Propósitos y Representaciones, 7(2). <https://doi.org/10.20511/pyr2019.v7n2.305>

Morrison, K.E.; DeBrabander, K.M.; Jones, D.R.; Ackerman, R.A. y Sasson, N.J. (2020). Social Cognition, Social Skill, and Social Motivation Minimally Predict Social Interaction Outcomes for Autistic and Non-Autistic Adults. Frontiers in Psychology, 11. <https://doi.org/10.3389/fpsyg.2020.591100>

Neuhaus, E.; Webb, S.J. y Bernier, R.A. (2019). Linking social motivation with social skill: The role of emotion dysregulation in autism spectrum disorder. Development and Psychopathology, 31(3), 931-943. $<$ https://doi.org/10.1017/s0954579419000361>

Pasion, R.; Dias-Oliveira, E.; Camacho, A.; Morais, C. y Campos Franco, R. (2020). Impact of COVID-19 on undergraduate business students: A longitudinal study on academic motivation, engagement and attachment to university. Accounting Research Journal [edición en línea previa a la publicación impresa]. <https:/doi.org/10.1108/arj-09-2020-0286> 
SaIde, S. y Sheng, M.L. (2020). Knowledge exploration-exploitation and information technology: Crisis management of teaching-learning scenario in the COVID-19 outbreak. Technology Analysis \& Strategic Management, 1-16.

<https://doi.org/10.1080/09537325.2020.1854714>

Sánchez-Bolívar, L.; Escalante-González, S. y Martínez-Martínez, A. (2021). Análisis motivacional del alumnado universitario transfronterizo de Ceuta y Melilla. Revista Complutense de Educación, 32(3), 405-414. $<$ https://doi.org/10.5209/rced.70306>

ShI, M. y Du, T. (2020). Associations of emotional intelligence and gratitude with empathy in medical students. BMC Medical Education, 20(1). <https://doi.org/10.1186/s12909-020-02041-4>

Shin, M. y Hickey, K. (2020). Needs a little TLC: Examining college students' emergency remote teaching and learning experiences during COVID-19. Journal of Further and Higher Education, 1-14. <https://doi.org/10.1080/0309877x.2020.1847261>

Sokolovskaya, I.E.; Polyakova, O.B.; Romanova, A.V.; Belyakova, N.V. y TereshCHUK, K.S. (n. d.). Educational and professional motivation of students with various religious orientations. European Journal of Science and Theology, 16(4), 169-180.

Trigueros, R.; Padilla, A.; Aguilar-Parra, J.M.; Lirola, M.J.; García-Luengo, A.V.; Rocamora-Pérez, P. y López-Liria, R. (2020). The Influence of Teachers on Motivation and Academic Stress and Their Effect on the Learning Strategies of University Students. International Journal of Environmental Research and Public Health, 17(23), 9089. <https://doi.org/10.3390/ijerph17239089>

Tseng, H.; YI, X. y YeH, H.-T. (2019). Learning-related soft skills among online business students in higher education: Grade level and managerial role differences in self-regulation, motivation, and social skill. Computers in Human Behavior, 95, 179-186. <https://doi.org/10.1016/j.chb.2018.11.035>

van der Stouwe, T.; Asscher, J. J.; Hoeve, M.; van der Laan, P. H. y Stams, G. J. J. M. (2018). The Influence of Treatment Motivation on Outcomes of Social Skills Training for Juvenile Delinquents. International Journal of Offender Therapy and Comparative Criminology, 62(1), 108-128. <https://doi.org/10.1177/0306624X16648130>

Vishkin, A.; Ben-Nun Bloom, P.; Schwartz, S.H.; Solak, N. y Tamir, M. (2019). Religiosity and Emotion Regulation. Journal of Cross-Cultural Psychology, 50(9), 1050-1074.

<https://doi.org/10.1177/0022022119880341> 